\title{
3D Knitted Preforms Using Large Circular Weft Knitting Machines
}

\author{
Christoph Peiner ${ }^{1}$ D $\cdot$ Henning Löcken ${ }^{1} \cdot$ Leon Reinsch $^{1} \cdot$ Thomas Gries $^{1}$
}

Received: 12 May 2021 / Accepted: 2 August 2021 / Published online: 26 October 2021

(c) The Author(s) 2021

\begin{abstract}
3D-knits are typically produced on flat knitting or special circular knitting machines in a time-consuming process. The utilisation of a sewing-like pattern opens up the potential of a 10-50 times faster production on common large circular knitting machines as compared to conventional flat knitting machines. Since common large circular knitting machines are not designed for this technology, the limitations and the stability of process conditions have been rarely studied. To further proof the feasibility and increase technological maturity, agile product development is conducted. As a concept product, a sports bra with customised cups is developed and demonstrated, which is knitted in a single step in approx. $5 \mathrm{~min}$ using the sewing-like pattern on a large circular knitting machine. The agile process is utilised to build process knowledge, develop a methodology for product design and to defined a descriptive process terminology. In order to further accelerate and digitize the production process, an algorithm is developed and implemented to convert CAD-data into machine readable knitting structures. The paper presents the basics of the 3D-Knit one large circular knitting machines and a defined terminology. Furthermore, process knowledge is built up and from this a sequence of development steps is derived. The development of a 3D-knitted sports bra is presented as a proof of concept of the technology. Further, the first steps for digitalising the product development process are given. The results open up application possibilities in sportswear, upholstery and aerospace with increased productivity and hence cost reduction.
\end{abstract}

Keywords Large circular 3d-knitting $\cdot 3 \mathrm{D}$ fabric $\cdot$ Productivity $\cdot$ Sewing pattern $\cdot$ CAD to pattern transformation

\section{Introduction-Definition 2D- and 3D-Textiles}

The transition between regular 2D-fabrics and 3D-fabrics can be defined in different ways and rarely there exists a strict border between 2D- and 3D-fabrics. The reason for this vague boundary is that all physical objects are formed in all 3 dimensions. At most, it happens that the expression of one dimension is considered to be infinitesimal compared to the

Christoph Peiner

christoph.peiner@ita.rwth-aachen.de

1 Institut Für Textiltechnik der RWTH Aachen University, 52074 Aachen, Germany 
other two. In conventional fabrics, the thickness is considered to be neglectable compared to width and length, hence these conventional fabrics are labelled as 2D-fabrics.

Fabrics which are referred to as 3D-fabrics have characteristic values in all 3 physical dimensions. The 3D-form of these textiles can be created due to the yarn architecture or the textile architecture. The yarn architecture is used to describe the composition of the yarns at the inside of a fabric. The fabric is defined as a 3D fabric due to its yarn architecture, if it is created by three or more yarn systems and no rectangular coordinate system can fit into it while maintaining a rectangular position to each yarn system. For example, spacer fabrics are typically considered 3D fabrics due to their yarn architecture.

The textile architecture is determined by the geometry of the fabrics. A fabric is defined as a 3D-fabric in regards to the textile architecture, if a volume is formed or created by the fabric; therefore, socks are typical regarded as 3D-fabrics [1,2].

The production of 3D-textiles can be carried out in a one-step-process or with a multiple-step process. Common technologies for multiple-step-processes are the production of $2 \mathrm{D}$-fabrics and sewing the textiles in their desired 3D-geometry or the pressure forming of elastic flat textiles into a 3D-geomety. For the one-step-process production of 3D-textiles various technologies are used, working mostly with the principles of fabric production from the conventional technologies for $2 \mathrm{D}$-fabrics. The most widely used 3D production technologies are 3D-braiding, 3D-warp knitting, 3D-weft knitting on flat knitting machines and 3D-weaving [1].

In this work, a new method is being developed to produce 3D-textile architectures on large circular knitting machines in a one-step-process. To date, spacer fabrics are the only 3D-textiles producible on common large circular knitting machines. 3D-textile architectures are typically produced on flat knitting machines or special seamless machines. Fuhrmann et al. previously developed a basic principle of forming 3D-textile architectures on large circular knitting machines [4-6].

\section{State of the Art - Principle of Forming 3D-Textiles on Large Circular Knitting Machines}

The principle of large circular 3D-knitting enables the production of 3D shaped knitted fabrics while still using the high productivity of the continuous needle movement of large circular knitting machines. There are no machine modifications on jacquard knitting machines needed and the productivity of large circular knitting machines can be enhanced with the new technology. As it is generally the case with 3D knitting, the prerequisite for this application is individual needle control, which means a jacquard machine is required [3-7].

The principle of producing 3D-knitted fabrics on large circular knitting machines is based on a new knitting pattern. With this pattern the implementation of a reduction of the surface, which is typically made by confection during the cut \& sew process, is enabled. Thus, the integration of these "sewing-like" knitting patterns (of darts) in various forms and positions results in a three-dimensional knitted form. The knitting pattern consists of floats and stitches that alternate horizontally over the area to be reduced (Fig. 1). Due to the continuous stitch wales of floats, the corresponding needles are not moved in this area and thus hold the knitted fabric in position. The other needles continue to form loops, but produce these on the backside of the knitted fabric. In this way, the continuous movement of the 
pixel image

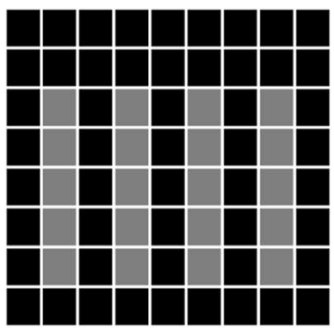

pixel scheme

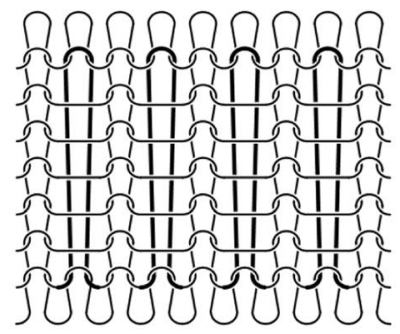

knitted fabric

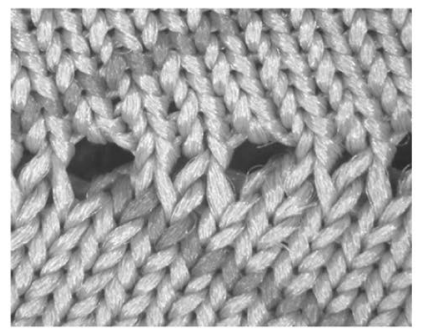

knitted loop

float

Fig. 1 Sewing like pattern for large circular 3D-knitting

circular knitting machines can be maintained and the effective surface area can be reduced [3-6].

Although the sewing like pattern enables the production of $3 \mathrm{D}$ knitted fabrics, it is not possible to achieve the same high flexibility of flat knitting machines. It is still necessary to cut the fabric out of the knitted tube and the reduced fabric remains on the backside (Fig. 2), and therefore does not reduce the amount of material used [3-6].

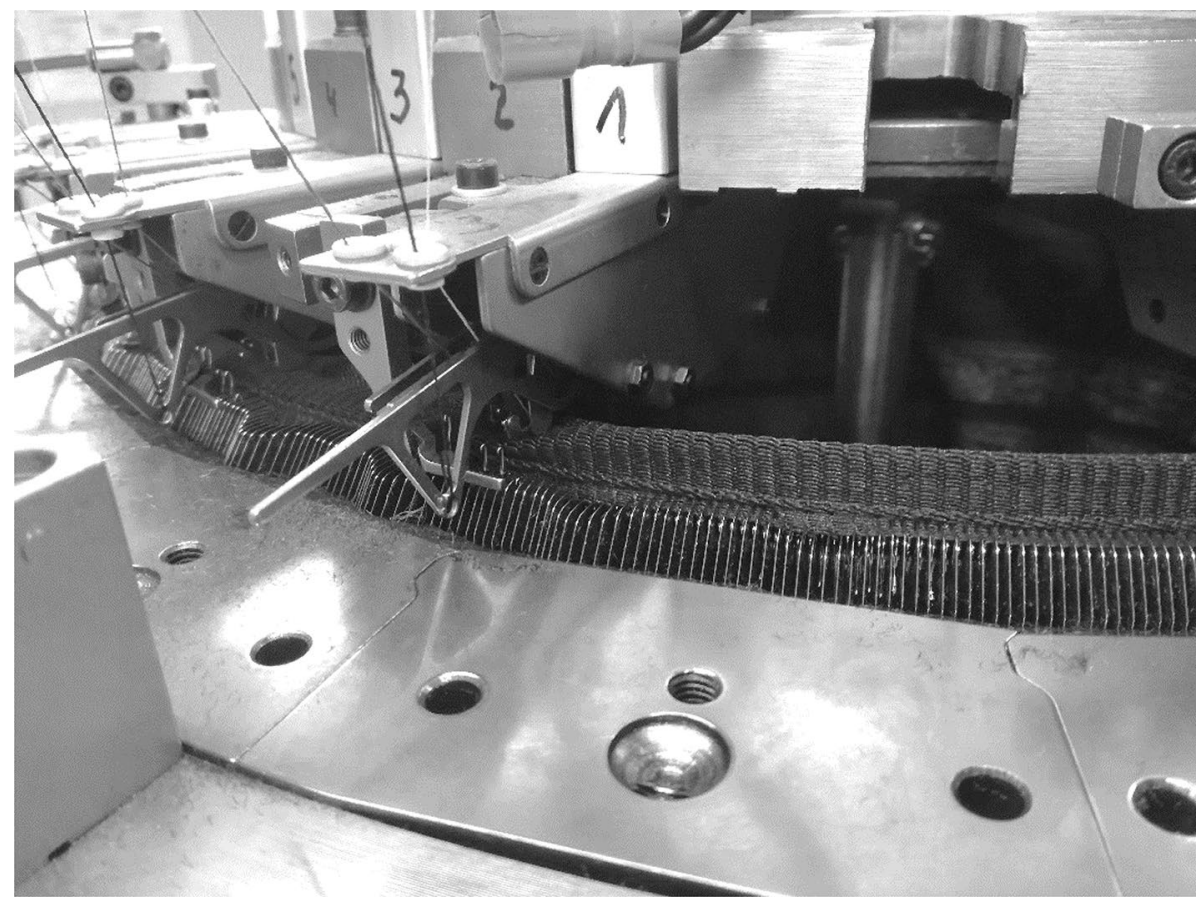

Fig. 2 Loop formation of 3D-Knit darts 


\section{Research Objective and Methodical Approach}

In this work, the principle of large circular 3D-knitting is further developed and limiting factors are being investigated. Therefore, important terms and characteristic parameters are defined, a complete product development cycle is developed and for the process step of 3D forming, an optional digital concept is developed.

In practical experiments, the relations between the shape, position and characteristic parameters of the structures with the resulting geometry are investigated. The experiments are conducted as a conceptual development of a product prototype - a Minimal Viable Product (MVP). During this product development, the most important influencing factors on the respective requirements of the sports bra are examined.

First, the requirements for a knitted sport bra are defined. To fulfil these requirements different possible knitting structures are investigated regarding their influence on the given requirements. Furthermore, important parameters in the design of the sewing-like pattern are examined for their influence on the size, shape and other properties. The findings of the experiments result in the identification and evaluation of characteristics concepts and elements. The most important of these characteristics are then defined and a descriptive terminology is developed.

The design process is further developed by investigation of a digital concept for the pattern generation with regard to location and size of sewing-like patterns.

\section{Development of a Sports Bra Using Large Circular 3d-knitting}

The development of a knitted sports bra includes complex requirement, engineering and optimisation of various product characteristics. For the investigation and further development of 3D knitting, only physiological properties of the sports bra are considered at this stage. Other properties, such as design, aesthetics or durability, are not in this scope. The physiological properties examined can be divided into three categories: thermological, skin sensory and ergonomic $[8,9]$.

Ergonomic comfort is determined by the fit of the fabric and the freedom of movement it allows during the usage. To obtain an ergonomic comfort in a sports bra, the freedom of movement must be reduced so that slipping of the sports bra is prevented, even during heavy physical activity. The skin sensorial comfort of a sports bra is determined by the haptics and the feel of the fabric on the skin. Since poor skin sensory comfort can lead to skin irritation, clothing should not scratch or stick to damp skin [10]. Thus, a high abrasion resistance and again the reducing of the freedom of movement are requirements for a sports bra. The thermological comfort of a sports bra is determined by its ability to support the thermoregulation of the body. The facilitation of rapid perspiration removal and good air permeability are beneficial [9]. A summary of the requirements for a knitted sports bra is given in Table 1.

Table 1 Overview of requirements for knitted sports bra

\begin{tabular}{lll}
\hline Thermological & Skin sensorial & Ergonomic \\
\hline High air permeability & High abrasion resistance & Fit of the fabric \\
Rapid perspiration removal & Low freedom of movement & Low freedom of movement \\
\hline
\end{tabular}


For rapid prototyping, a first fabric with basic fit is needed for further development and optimisation. A "cutting pattern" forms the basis for this and has major impact on skin sensory and ergonomics. On top of that, different patterns enable further functionalisation of different areas. A physical prototype helps to identify needs for optimisation and to conduct segmentation of product and pattern.

To develop a first prototype, a mannequin is measured and its body shape is moulded by hand. The moulding helps to generate the first iteration of the knitted pattern. This moulding method is known from the classic clothing industry and is suitable for transferring the shape of an object into a textile construction. In analogy to darts in garment technology, triangle forms are also used for area reduction in large circular 3D knitting. The further fit is then iteratively checked and evaluated by knitting and pulling the $3 \mathrm{D}$ knit onto the mannequin. The iterative optimisations include the reduction or enlargement of the sewing like pattern in height and width as well as adjustments of the exact positioning of the sewinglike patterns.

One limitation of 3D knitting is the orientation of the sewing-like pattern. In principle, they can be positioned freely, but the area reduction can only be made in course direction. For an area reduction in wale direction, the transfer of the stitches to adjacent needles would be necessary. This function has not yet been realised in conventional large circular knitting machines and only exists in flat knitting machines. Thus, it is necessary to determine at the beginning of the development process in which main direction the sewing-like patterns will be arranged (Fig. 3). In this stage, the orientation within the production direction is chosen for further development, to support the posture of the breasts.

In order to enhance the body fit, the regular 2D knitted patterns can be adjusted to bring more stretch or stiffness into certain areas. To investigate which knitted structures cause higher stretch in the textile, tests were carried out on normal 2D structures. For

\section{Sports bra oriented perpendicular to the production direction}

\section{Sports bra oriented within the production direction}
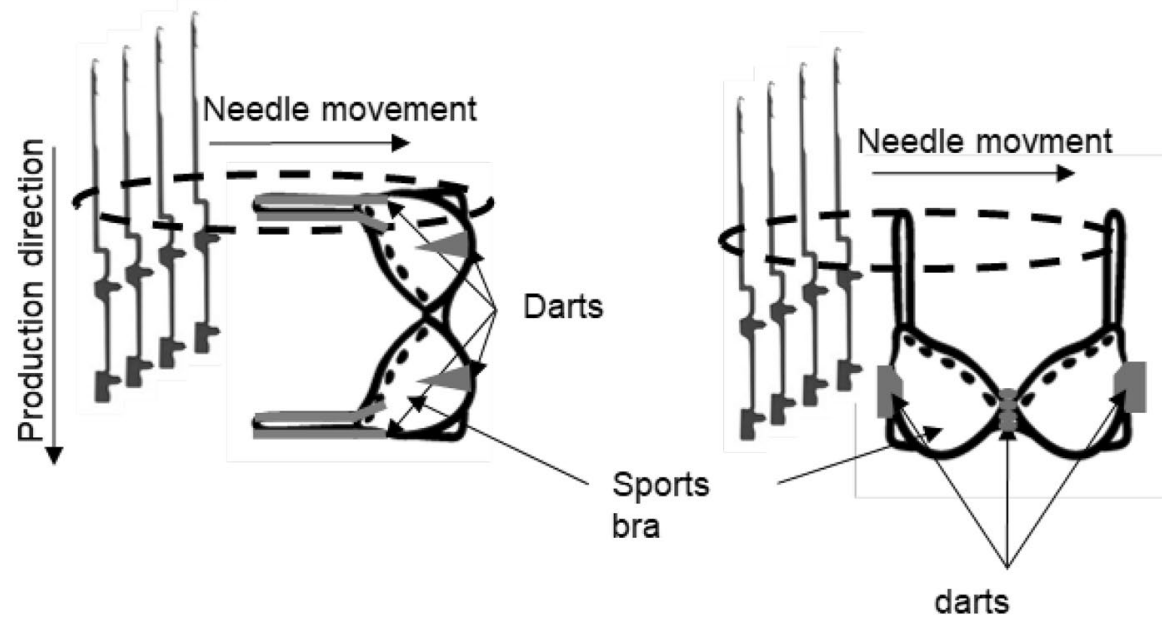

Fig. 3 Orientation of sports bra regarding the production direction 
Table 2 Knitting pattern amount of loops, tucks and floats

\begin{tabular}{llll}
\hline Knitting pattern & amount of loops & amount of tucks & amount of floats \\
\hline Single Jersey & $100 \%$ & $0 \%$ & $0 \%$ \\
Pique & $75 \%$ & $25 \%$ & $0 \%$ \\
Maglia Spiga & $50 \%$ & $50 \%$ & $0 \%$ \\
Locknit & $75 \%$ & $0 \%$ & $25 \%$ \\
CrossMiss & $50 \%$ & $0 \%$ & $50 \%$ \\
\hline
\end{tabular}

these tests, five different knitted structures were produced and analysed in the test laboratory (Table 2). The knitting structures differ in their proportion of tucks and floats. Furthermore, two process parameters, yarn tension and knock over depth, were varied.

To analyse the stretch, the knitted structures were tested in a tensile test. The tensile tests were carried out according to DIN EN ISO 13,934-2. In addition to the tensile tests, air permeability tests were also carried out according to DIN EN ISO 9237. Figure 4 shows that the proportion of floats and the knock-over depth in particular have a strong influence on both the air permeability and the stretch of a knitted fabric. In order to support the formation of the shape in special areas with a higher stretch of the knitted fabric, it is useful to use the knitting pattern CrossMiss. Unfortunately, it is not possible to change the knock-over depth during the process on conventional large circular knitting machines.

For the final production of a sport bra, it is necessary to cut the fabric out of the knitted tube. The cutting is necessary to create the arm, head and body holes but a cut and sew process to achieve the body-fitting 3D-shape is no longer necessary. The backside material of the sewing-like pattern is used as a fitting material, since this backside material has a softer texture than the standard knitted fabric. With the reduction of some of these production steps after the knitting, and with a short knitting time of around 5 min, a higher productivity, as compared to flat knitting and the classic confection, can be obtained with the large circular 3D-knitting (Fig. 5).
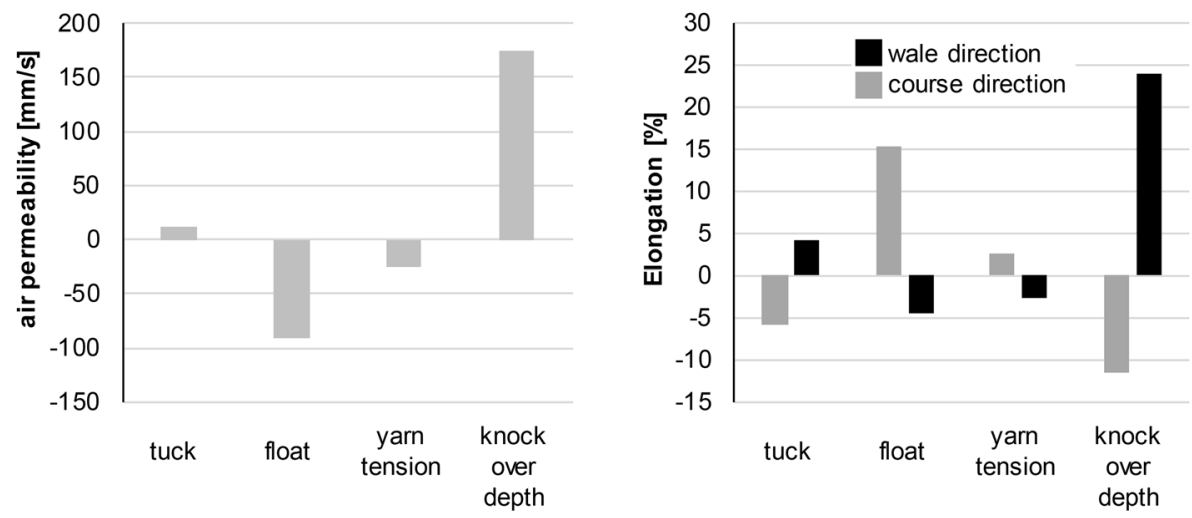

Fig. 4 Influence of tuck and floats onto air permeability and elongation 


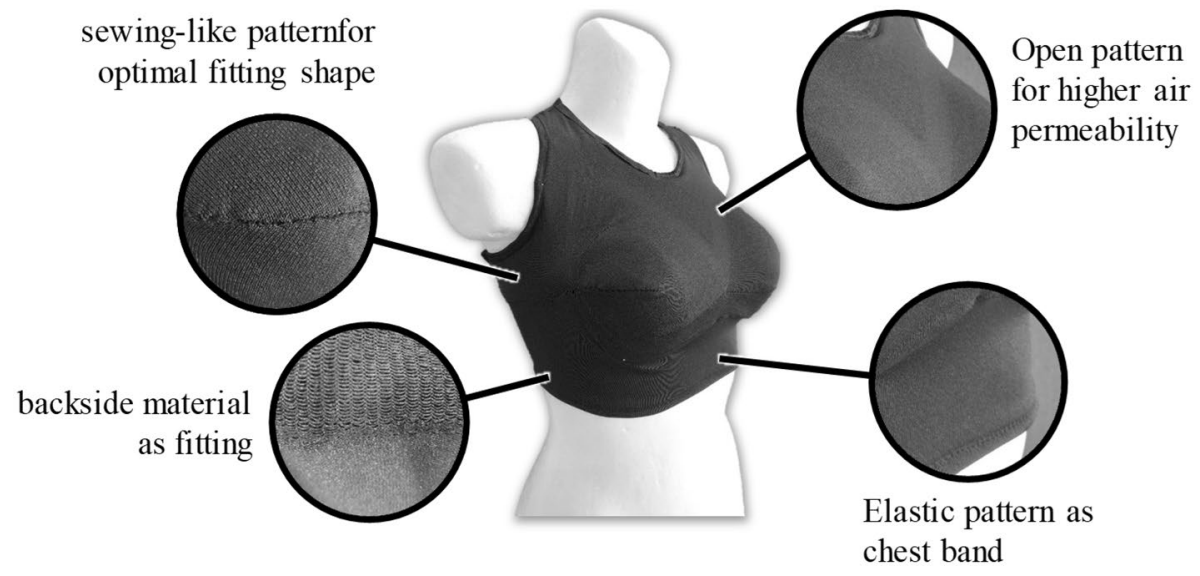

Fig. 5 3D-knitted sports bra produced on large circular knitting machine

\section{Evaluation of the Development Process}

Agile development process led to quick iterations and helpful insights. Findings and assumptions have to be collected during the process, evaluated and utilised for the development of a process methodology. Throughout the development of the knitted sports bra on the large circular knitting machine, various observations were made. To create the first prototype, it is still necessary to manually mould the desired geometry and then transfer it into a machine program. During the first development steps it is necessary to determine the direction of production and the knock over depth, since both parameters can only be changed by starting a new development process.

After the determination of these two variables the form and position of the sewing-like patterns were conducted. Although it is possible to adjust the sewing-like patterns at a later stage, it makes practical sense to determine the position and shape as early as possible. Especially if it is necessary or beneficial to use different knitting patterns to enhance the products properties like air permeability. Due to an influence of these patterns onto the geometry, the interaction makes it difficult to change the sewing-like patterns in the late course. A collection of the further findings is given in Table 3.

\section{Terminology}

The shape-forming element in the large circular 3D-knitting is the characteristic sewinglike pattern. The pattern is integrated as a geometric form into the pixel image that is used as a basis for the knitting program. In line with the reduction of surface area in garment production, triangles are usually used as basic shapes for the sewing-like pattern in product development (Fig. 1). By using different shapes and by varying different parameters when creating this geometric form, the three-dimensional effect and the appearance can be adjusted within wide limits. In the following, the parameters are defined and the terminology is explained. The parameters are divided into the following three categories: 


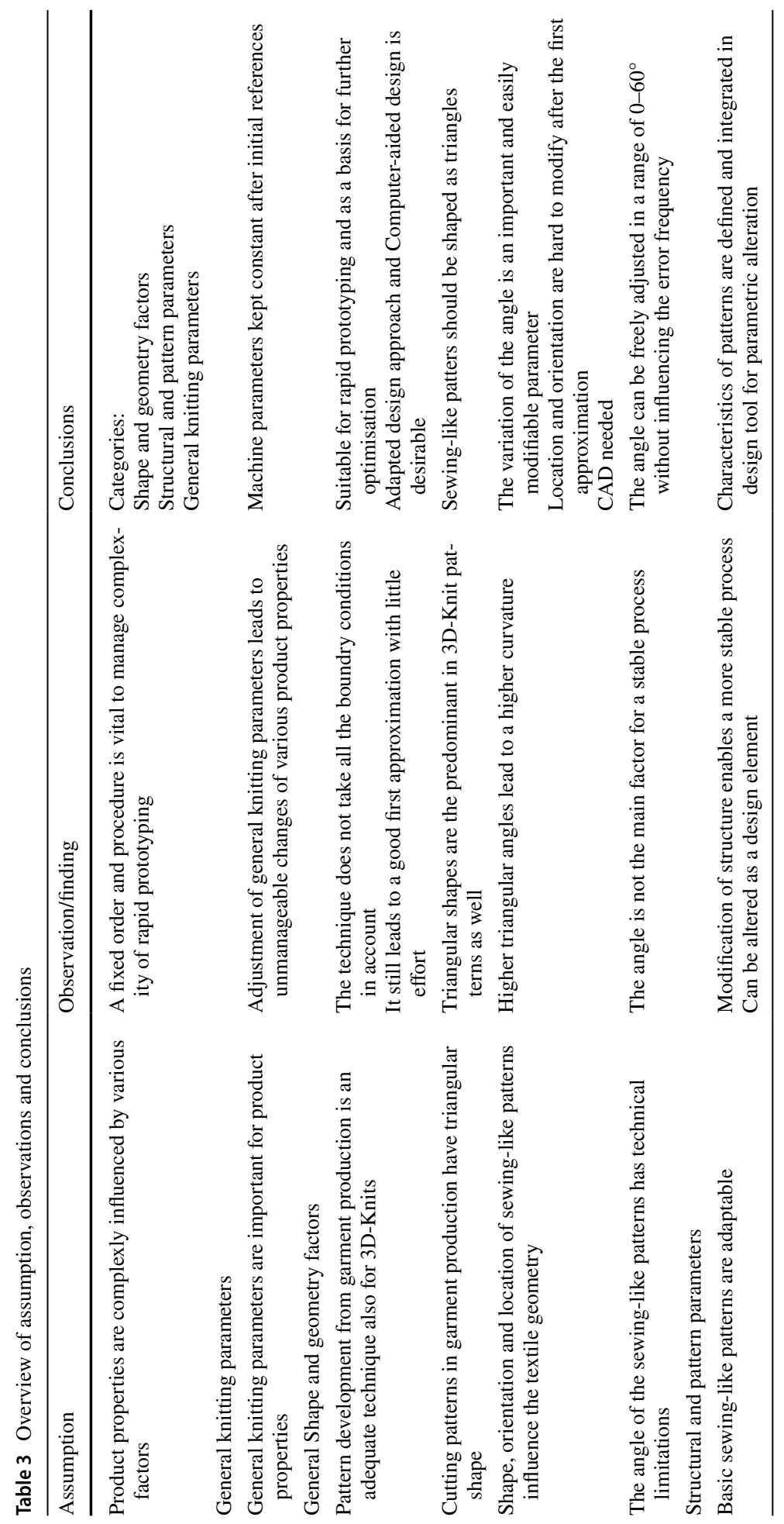




\section{Segment length $I_{s}$}

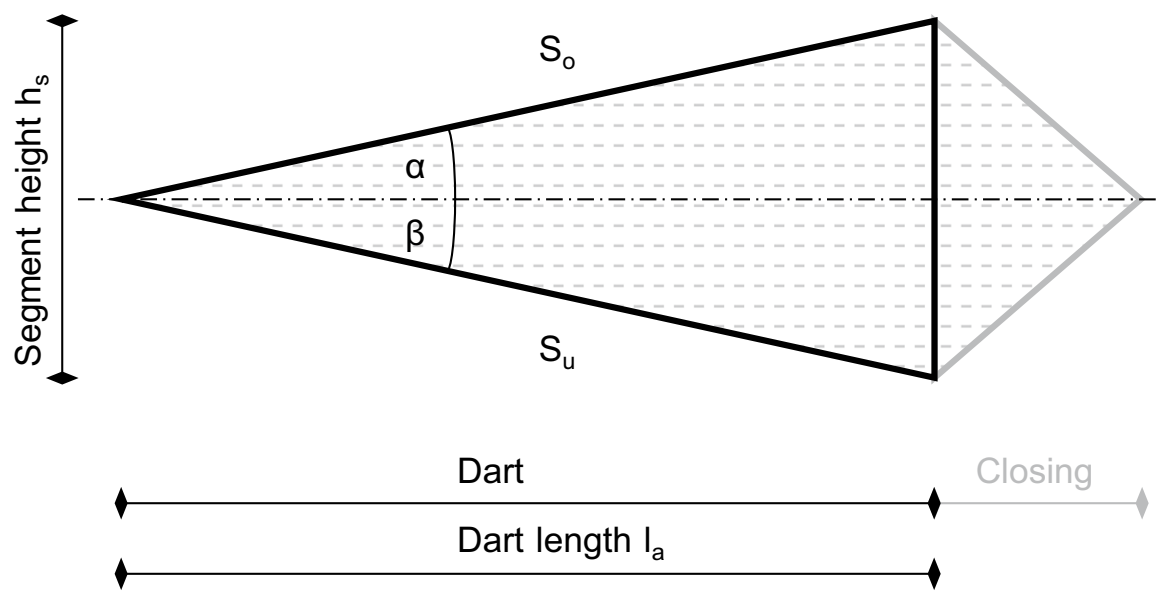

Fig. 6 Overview of dart shape parameters

- Shape and geometry factors

- Structural and pattern parameters

- General knitting parameters

Due to the predominant use of triangles as pattern geometries, the shapes are exclusively considered as triangles in this section. The shape and geometry parameters are defined on the basis of the dart triangle (Fig. 6) but can be applied analogously for other geometric shapes. Only the part of the entire system required for shaping is considered as the shape building pattern (black lines). In addition, a closing can be added to the overall system. The closing is not meant to influence the shaping. For an even knitted surface and process stability/risk mitigation, closing has been considered useful up to now.

Table 4 gives an overview of the terms for the elements of the ITA 3D-Knit technology and their definition. The size of a typical triangular dart is described by the darts height and length. The dart height corresponds to the segment height for triangular darts. The dart angle can be calculated directly from the dart height and length for a straight knitting line. In the case of curved knitting lines, the dart angle is measured between the pitch tangent of the knitting line and the (imaginary) centre line of the dart. By increasing the dart angle, a stronger curvature and a higher 3D effect is achieved with large circular 3D-knitting. The upper and lower halves of a dart do not necessarily have to be symmetrical. In principle, completely different shapes are also possible here. In the development so far, mainly symmetrical darts have been used.

In variation to triangles with straight edges, the sewing-like patterns can also be realised with different knitting lines. Typical shapes used for the knitting lines are concave or convex curves or staircase functions. The influence of a dart on the shaping can be influenced to a small extent by the alternative knitting lines. The shapes of the knitting lines have a major influence on the appearance of the dart. 
Table 4 Terms and Definition for the Elements of large circular 3D-knitting

\begin{tabular}{|c|c|}
\hline Term & Definition \\
\hline Segment & - Whole area of the sewing-like pattern, including the dart and the closing \\
\hline $\begin{array}{l}\text { Darts / } \\
\text { Sewing-like }\end{array}$ & $\begin{array}{l}\text { - Main part of the segment, that is designed for the 3D-shaping. } \\
\text { - Can be identical to the segment, if the closing is designed for shaping as well. }\end{array}$ \\
\hline Closing & $\begin{array}{l}\text { - The part of the segment, that is designed to support a smooth closing and preventing an } \\
\text { abrupt exit. }\end{array}$ \\
\hline Dart length & - Length of the dart, measured in pixels/stitches \\
\hline $\begin{array}{l}\text { Closing } \\
\text { length }\end{array}$ & - Length of the closing, measured in pixels/stitches \\
\hline $\begin{array}{l}\text { Segment } \\
\text { length }\end{array}$ & $\begin{array}{l}\text { - Length of the segment, measured in pixels/stitches } \\
\text { - Equals dart length }+ \text { closing length }\end{array}$ \\
\hline $\begin{array}{l}\text { Segment } \\
\text { height }\end{array}$ & - Height of the segment, measured in pixels/stitches \\
\hline Dart angle & $\begin{array}{l}\text { - Angle between the gradient tangent at the knit line and the (possibly imaginary) center } \\
\text { line }\end{array}$ \\
\hline Center line & $\begin{array}{l}\text { - Horizontal knit line in the center of a segment } \\
\text { - Consists only of stitches and divides the dart into two waves }\end{array}$ \\
\hline
\end{tabular}


Table 4 (continued)

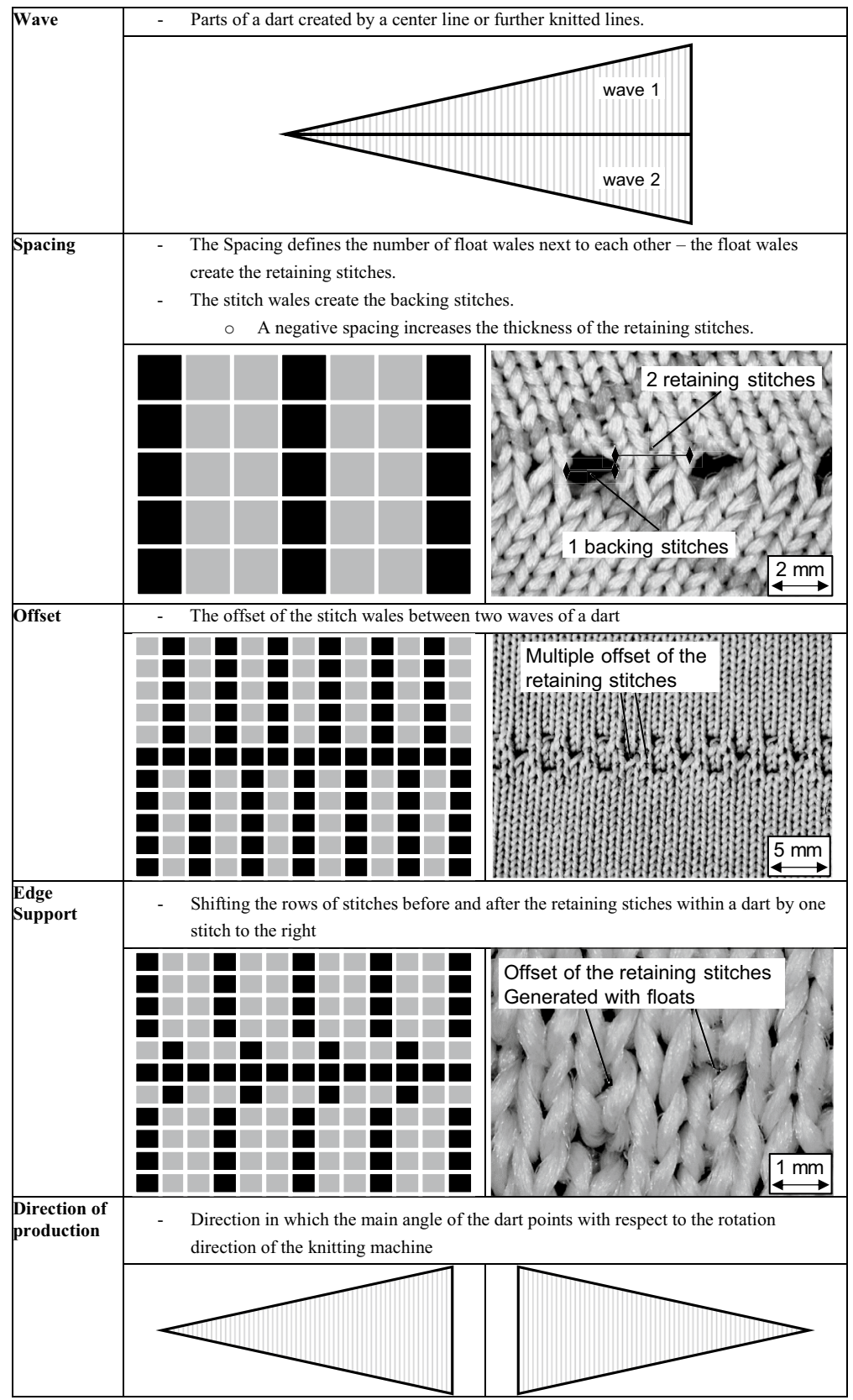

In addition to the shape and geometry factors, there are other influencing variables that have a particular impact on the appearance and process reliability of the sewing-like pattern. The structural and binding parameters are the arrangement or variation of the binding elements stitch and float. The basic sewing-like pattern consists of alternating wales of 
floats and stitches. This basic pattern can be altered using different binding parameters. The currently observed binding parameters are:

- Number of waves

- Spacing

- Offset

- Edge Support

The number of waves of a dart indicates how many partial darts (waves) a dart consists of. By inserting a centre line, the dart is divided into two partial dart sections. The centre line consists only of stitches to divide the dart. This row of stitches additionally anchors the knitted fabric in the centre of the dart on the backside of the fabric. By inserting more horizontal stitch lines, a dart is further divided and several, smaller waves are created.

Spacing describes the number of float wales arranged next to each other. With a continuous alternation of stitch and float wales with a width of one pixel, the spacing is 1 . If the width of the float wales is increased, and the spacing is the number of pixels in the width of the float wale. If, on the other hand, the width of the knitting stitch is increased, the spacing is displayed in the negative range. A dart with stitch wales with a width of 2 pixels has the spacing -2 . The spacing -1 corresponds to the same structure as the spacing 1 .

The so-called holding stitches are created from the float stitches of the dart in the real knitted fabric. In a dart, the so-called backing stitches are created from the stitch bars of the pixel image. The offset is the offset of the pixels in the stitch wales between two waves. With an offset of 1 , the repetition sequence of stitch and float is shifted one pixel to the right. The offset can only be used with a wave count of 2 or more.

Another variation of the binding parameters is the Edge Support. With the Edge Support, the rows of stitches before and after a knit line are shifted by one pixel. This shift only takes place on horizontal knit lines. Furthermore, the conventional parameters of the knitting process also have an influence on the appearance of the darts. The most important knitting parameters in connection with the darts are the stitch length, the yarn tension, the machine gauge and the yarn count.

\section{Automated Digital Pattern Construction}

The advances in 3D textiles and especially the presented ITA 3D-Knit technology require new tools, processes and computer-aided support to bridge the gap between design ideas and technical realisation $[2,11]$. Concerning the transfer of 3D shapes into machine readable knitting instructions, much effort is directed towards flat knitting machines [12-15]. Due to technical limitations, the ITA 3D Knit technology can neither use the CAD systems for the garment and fashion industry nor the algorithms for flat knitting machines. While knitting elements in flat knitting machines can influence the textile dimensions both in wale and course direction, the ITA 3D Knit technology is only able to influence the structures in the course direction. Therefore, the presented approach addresses the boundaries or limitations of ITA 3D-Knit technology. The basis for the developed algorithm is the assumption that wales in two-dimensional knitted structures can be represented by parallel lines. The transition from Euclidean geometry in 2D to Non-Euclidean geometry leads to 
the mathematical and technical challenges in the design of 3D products. To assure the compliance with this technical boundary condition on three-dimensional complexly curved surfaces, the path can be calculated by means of differential geometry. The algorithm makes use of the parametric equations of surfaces and curves in $\mathbb{R}^{3}$, normal vectors and tangent planes [16].

$$
\begin{gathered}
F(x, y)=\left(\begin{array}{l}
x \\
y \\
z
\end{array}\right), \text { with } x, y \in \mathbb{R} \\
\vec{n}= \pm\left(\begin{array}{c}
-\partial F / \partial x \\
-\partial F / \partial y \\
1
\end{array}\right) \\
\gamma(t)=\left(\begin{array}{l}
x \\
y \\
z
\end{array}\right), \text { with } t \in I, I=[0,1] \\
\frac{t(t)=\frac{\dot{\gamma}(t)}{|\dot{\gamma}(t)|}}{\overrightarrow{t_{M R R}}}= \pm \overrightarrow{t_{M S R}} \times \frac{\vec{n}}{|\vec{n}|}
\end{gathered}
$$

Figure 7 illustrates the basic numeric steps (c) from the parametric surface representation (a) to the polygon mesh (b). With the Eqs. (1)-(5) in the first step the path of each wale is mapped to the surface. In the second step, the wales are connected in course direction. Since the ITA 3D-Knit allows for manipulation in course direction, this step is carried out rule-based, similar to presented approaches for flat knitting machines [15]. Locally, the intersections between course and wale direction should be as orthogonal as possible. If the curvature of the surface leads to too much distortion and deviation from the orthogonality, this must be regulated by the use of the sewing-like pattern. In the last step, the resulting network is transformed to a $2 \mathrm{D}$ pattern.

As a final result, this automated algorithm transforms a given surface in a $2 \mathrm{D}$ pattern. The $2 \mathrm{D}$ pattern is suitable for compilation to machine readable knitting instructions. The final instructions are knittable on large circular knitting machines. With the help of the algorithm, the sectional image of a hemisphere is created. Visual inspections confirm the resemblance of the resulting structure with the targeted surface.

In future work, the aim will be to calculate the paths in wale direction more efficiently. The computation of parallel curves on parametric surfaces is a suitable approach [17]. The approach allows for variation of stitch dimensions and can therefore include different knitting structures within one product. However, the lack of mathematical representations of three-dimensional surfaces in industrial applications may require the methods and algorithms of discrete differential geometry in future improvements [18]. 

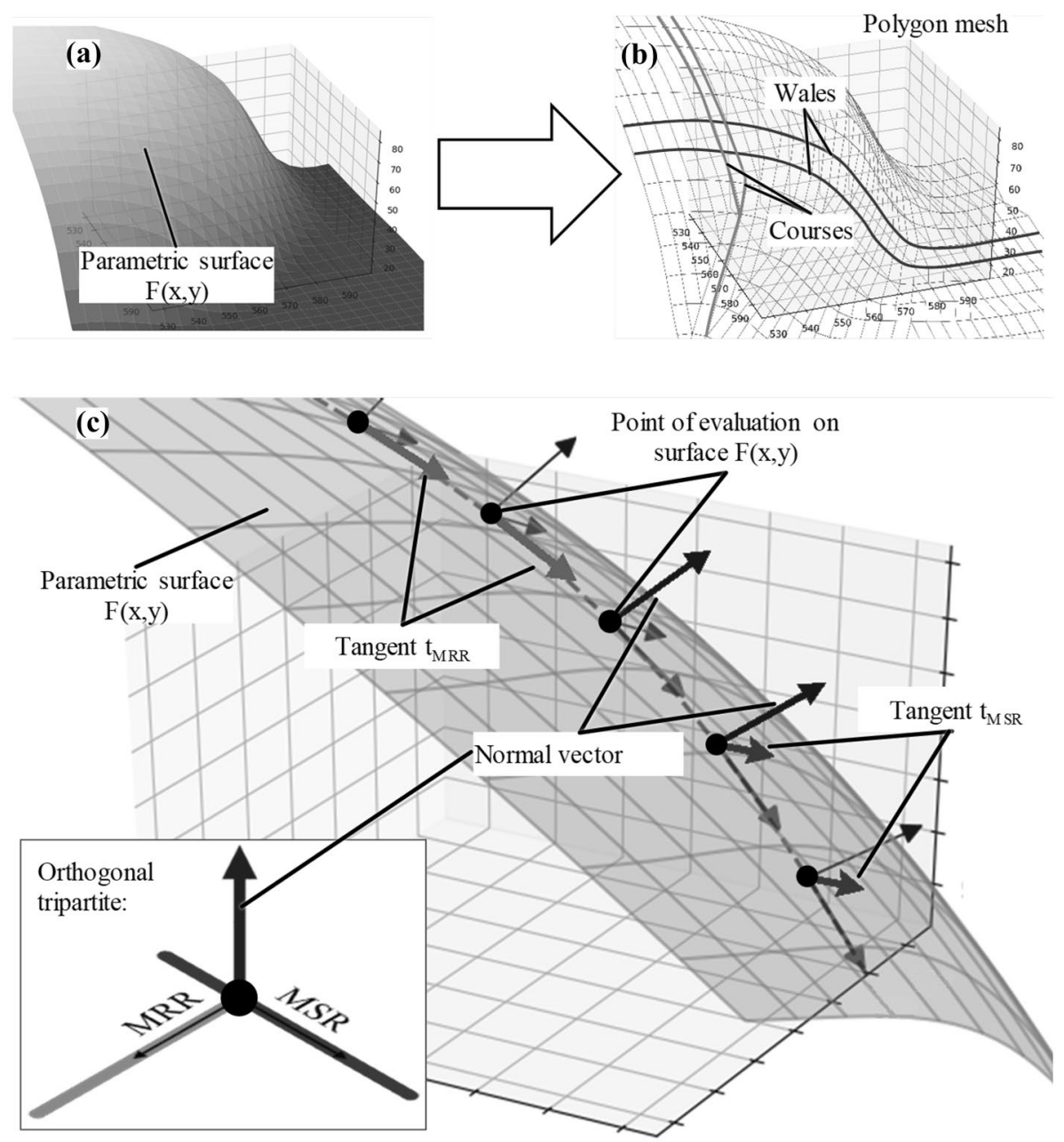

Fig. 7 Parametric surface (a), calculated polygon mesh (b) and basic steps of developed algorithm (c)

\section{Conclusion and Future Outlook}

The new developed ITA 3D-Knit Technology enables the production of 3D knitted fabrics on large circular knitting machines. The proof of concept is established using a 3D knitted sports bra. The sports bra shows the immense possibilities of the ITA 3D-Knit technology. By using large circular knitting machines, the productivity for 3D-knitted textile is increased significantly. The knitting process of the sports bra described above only takes around $5 \mathrm{~min}$. With the use of Jacquard knitting machines, it is further possible to insert various knitting patterns for thermoregulation, design applications or higher comfort.

Although the ITA 3D-Knit Technology provides a higher productivity for 3D-knitted fabrics, it is not possible to achieve the same high flexibility compared to flat knitting machines. Within the ITA 3D-Knit Technology, it is still necessary to cut the fabric out of the knitted tube and to finish the edges. 
A further challenge is the finishing of 3D fabrics. The standard processes used in the textile industry such as washing in a padder and heat-setting in stenter are not applicable due to the 3D form and the varying dimensions of the fabrics. In order to meet the same quality requirements demanded for classical 2D textiles, it is necessary to develop new finishing processes for 3D textiles. A shape-retaining fixation is necessary for 3D textiles, so that the garment is not subjected to free shrinkage, which leads to strong fluctuations in the dimensions or has to be determined in extensive trials. In order to reduce the extent of this trial and to keep the shape within lower tolerances, a shape-retaining fixation on stiff fixation moulds is necessary.

In order to further advance the ITA 3D-Knit Technology, new product developments and innovations are necessary. With these new product innovations, the wide application of the technology and the high speed in the production of 3D knitted fabrics can be brought into the market. Promising approaches for the development of new products are the utilisation of the backside material as lining or filling fabric. To fully utilise the potential of 3D-knitted fabrics, the development of the digitalisation of the pattern development can be a key factor. For this purpose, the approaches investigated so far must be reviewed and the most promising algorithms have to be identified. With a strong correlation of these mathematical algorithms and the textile characteristics, the digitalisation can lead to a significant reduction of the development costs for $3 \mathrm{D}$ fabrics.

Acknowledgements The authors would like to thank the several funding agencies supporting these various research topics. These include but are not limited to the German Federal Ministry of Economics and Technology (Bundesministerium für Wirtschaft und Technologie), German Federal Ministry of Education and Research (Bundesministerium für Bildung und Forschung) and the European Union.

Funding Open Access funding enabled and organized by Projekt DEAL. The various research topic were funded by several agencies. These include but are not limted to the German Federal Ministry of Economics and Technology (Bundesministerium für Wirtschaft und Technologie), German Federal Ministry of Education and Research (Bundesministerium für Bildung und Forschung) and the European Union.

Data Availability, Material, and Code The data, material and code can be found at the following contacts: Christoph Peiner, email: christoph.peiner@ita.rwth-aachen.de

\section{Declarations}

\section{Conflicts of Interest None.}

Open Access This article is licensed under a Creative Commons Attribution 4.0 International License, which permits use, sharing, adaptation, distribution and reproduction in any medium or format, as long as you give appropriate credit to the original author(s) and the source, provide a link to the Creative Commons licence, and indicate if changes were made. The images or other third party material in this article are included in the article's Creative Commons licence, unless indicated otherwise in a credit line to the material. If material is not included in the article's Creative Commons licence and your intended use is not permitted by statutory regulation or exceeds the permitted use, you will need to obtain permission directly from the copyright holder. To view a copy of this licence, visit http://creativecommons.org/licenses/by/4.0/.

\section{References}

1. Roye, A., Stüve, J., Gries T.: Definition for the differentiation of 2-D and 3-D textiles, Technical Textiles $4 / 2005$ (2005)

2. Hearle, J.W.S.: Definition of 3D textiles in: X. Chen - Advances in 3D Textiles, Cambride: Woodhead Publishing Limited (2015) 
3. Fuhrmann K.: Erhöhung der Flexibilität der Großrundstricktechnologie zur effizienten Fertigung zweiund dreidimensionaler gestrickter Produkte im Sinne des Mass-Custamization-Ansatzes, Dissertation, RWTH Aachen University (2018)

4. Simonis, K., Gloy, Y.S., Gries, T.: 3D knitting using large circular knitting machines, 17th World Textile Conference AUTEX 2017: Shaping the Future of Textiles, 29-31 May 2017, Corfu, Greece. - IOP Conference Series: Materials Science and Engineering, 254 (2017)

5. Simonis, K., Gloy, Y.S., Gries, T.: 3D knitting using large circular knitting machines, In: The Fiber Society (Ed.): The Fiber Society 2017 Spring Conference: Next Generation Fibers for Smart Products, May 17-19, Aachen, Germany (2017)

6. Simonis, K., Gries, T.: 3D formed knitted fabrics by large circular knitting machines, Proceedings of the 8th World Conference on 3D Fabrics and Their Applications, Manchester, UK, 28-29 (2017)

7. Trümper, W.: Gestrickte Halbzeuge und Stricktechniken, In: Cherif C Textile Werkstoffe für den Leichtbau - Techniken - Verfahren - Materialien - Eigenschaften Berlin: Springer, p. 225-263 (2011)

8. Kirstein, T.: Produktentwicklung für körpernahe Bekleidung unter Berücksichtigung der textilen Materialeigenschaften Dresden, TU Dresden, Dissertation, (2000)

9. Mecheels, J.: Körper-Klima-Kleidung: wie funktioniert unsere Kleidung? Fachverlag Schiele \& Schoen, Berlin (1998)

10. Harnisch, M.: Sport und Komfort - Widerspruch oder vereinbar? - Bönnigheim, 2012, https://www. hohen-stein.de/de/inline/pressrelease_20609.xhtml, Zugegriff am (2019)

11. Bendt, E.: Shape and Surface: The challenges and advantages of 3D techniques in innovative fashion, knitwear and product design, 48th Conference of the International Federation of Knitting Technologists (IFKT), (2016)

12. Yuksel C., Kaldor, J., James, D., Marschner, S.: Stitch Meshes for Modeling Knitted Clothing with Yarn-level Detail. ACM TOG 31(3) - SIGGRAPH, (2012)

13. Wu, K., Swan, H., Yuksel, C.: Knittable Stitch Meshes. ACM Trans. Graph. 36(4), (2018)

14. Wu, K., Gao, X., Ferguson, Z., Panozzo, D., Yuksel, C.: Stitch Meshing. ACM Trans. Graph. 37(4), (2018)

15. Popescu M., Rippmann M., Van Mele T., Block P.: Automated Generation of Knit Patterns for Nondevelopable Surfaces. In: De Rycke K. et al. (eds) Humanizing Digital Reality. Springer, Singapore, (2018)

16. Bärwolff, G.: Höhere Mathematik für Naturwissenschaftler und Ingenieure. Berlin, Heidelberg: Springer Berlin Heidelberg, (2017)

17. Gálvez, A., Iglesias, A., Puig-Peytitle, J.: Computing parallel curves on parametric surfaces. Appl. Math. Model. 38(9), 2398-2413 (2014)

18. Sharp N., Crane, K.: You can find geodesic paths in triangle meshes by just flipping edges. ACM Trans. Graph. 39(6), Article 249, 15 pages, (2020)

Publisher's Note Springer Nature remains neutral with regard to jurisdictional claims in published maps and institutional affiliations. 